$$
\text { DOE/ER/40150--1212 }
$$

\title{
0.1 Beam Dynamics Activities at the Thomas Jefferson National Accelerator Facility (Jef- ferson Lab)
}

David R. Douglas

douglas $@ j l a b . o r g$

Jefferson Lab 12000 Jefferson Avenue $P$ Newport News, VA 23606, Nov 19199 ? USA

The Thomas Jefferson National Accelerator Facility (Jefferson Lab) has been funded by the US Navy to build an infra-red FEL driven by an energyrecovering compact SRF-based linear accelerator [1]. The machine is to produce a $1 \mathrm{~kW}$ IR photon beam. The Jefferson Lab Accelerator Division is presently engaged in detailed design and beam dynamics studies for the driver accelerator.

The accelerator base parameters are as follows:

Injection kinetic energy:

Beam kinetic energy at wiggler:

Beam kinetic energy at dump:

Beam current $(\mathrm{cw})$ :

Normalized RMS emittance design: nominal:

RMS momentum spread at wiggler:

Full momentum spread after wiggler:

FEL extraction efficiency:
$10 \mathrm{MeV}$

$42 \mathrm{MeV}$

$10 \mathrm{MeV}$

$5 \mathrm{~mA}$

$13 \mathrm{~mm}-\mathrm{mrad}$

$5 \mathrm{~mm}-\mathrm{mrad}$

$0.5 \%$

OSTI

Figure 1: IR-FEL Driver Accelerator Concept

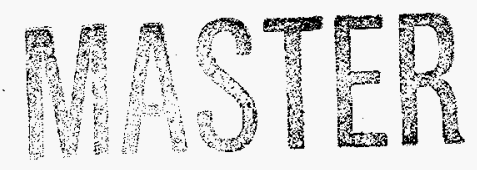

The machine concept (Figure 1) comprises a $10 \mathrm{MeV}$ injector based on a photocathode gun and a high-gradient Jefferson Lab accelerator cryounit, a 


\section{DISCLAMVER}

Portions of this docoment may be illegible in electronic imsge products. Ingges are produced from the best arailable original docomerert 
linac based on a single high-gradient Jefferson Lab accelerator cryomodule, the FEL itself, and an energy-recovery recirculation arc. Energy recovery is implemented as a means of limiting cost and technical risk by reducing the $\mathrm{RF}$ power requirements in the driver accelerator; following lasing, the beam is recirculated to the cryomodule and reinjected 180 degrees out of phase, so as to drive the SRF cavities using the power of the "spent" electrons. Following decceleration to $10 \mathrm{MeV}$, the beam is dumped.

Principle beam dynamics and beam transport considerations include:

- Generation and transport of a high-quality, high-current, space-charge dominated beam,

- The impact of coherent synchrotron radiation (CSR) during beam recirculation transport,

- Low-loss transport of a large momentum spread, high-current beam,

- Beam break up (BBU) instabilities in the recirculating accelerator,

- Impedance policing of transport system components, and

- RF drive system control during energy recovery and FEL operation.

To minimize beam quality degradation by CSR and space charge during transport to the FEL, the wiggler is placed immediately downstream of the linac. This reduces the impact of these collective effects, as only a chicane is encountered upstream of the laser, and allows initial low-power "first light" operation of the laser prior to the commissioning of the energy recovery transport.

The transport system concept is based on the successful large-acceptance recirculator at the MIT-Bates Accelerator Laboratory [2]. Following the cryomodule, a six-quadrupole telescope images the $42 \mathrm{MeV}$ electron beam to match the betatron acceptance of the wiggler. A four-dipole "dogleg", or chicane, embedded in the telescope displaces the electron beam around the end components of the FEL optical cavity, and then returns it to the wiggler/optical cavity axis. The momentum compaction of this chicane allows 
compression of the bunch length to picosecond time scales, increasing the peak current to enhance the FEL interaction. Downstream of the wiggler, a second quadrupole telescope (with embedded dogleg, to avoid the other end of the optical cavity) matches the electron beam to the betatron acceptance of the energy-recovery recirculation arc. The beam is transported through a linearly achromatic Bates-type end loop, through a FODO backleg, and into a second end loop to the reinjection point at the cryomodule.

The recirculation transport is nominally is isochronous from wiggler to reinjection point. Two families of trim quadrupoles allow variation of the $(5,6)$ matrix element between $-0.25 \mathrm{~m}$ and $+0.25 \mathrm{~m}$ while maintaining achromaticity; this dynamic range will allow optimization of the RF system behavior and energy compression of the electron beam during FEL operation with energy recovery. The required 5acceptance has been achieved through careful attention to chromatic aberrations. Such aberrations have been controlled through careful selection of hardware parameters (bend angles, entry/exit angles, and radii), suppressed by use of transport with rational tunes (the FODO transport comprises six cells with 90 degrees tune each; the end loops each are 1.25 wavelengths long horizontally and 0.5 wavelengths long vertically), and corrected using eight sextupoles (in two symmetrically arrayed families to reduce to zero at the reinjection point the quadratic variations with momentum of path length, horizontal position, and horizontal angle).

Beam dynamics studies using CEBAF-benchmarked methods predict BBU thresholds in excess of 60 milliamps [3]. Initial theoretical studies of CSR effects suggest that phase-space degradation at the wiggler will not preclude FEL operation; ongoing investigations are considering the impact of CSR during the energy recovery transport [4]. Modeling of space-charge effects during transport and acceleration using PARMELA indicate that beam quality is maintained through the acceleration cycle and the proper phase space for FEL operation can be produced at the wiggler; space charge does not significantly influence the beam performance once the final energy of $42 \mathrm{MeV}$ has been achieved[5]. Lattice design and analysis studies predict that machine beam transport parameters are readily achieved and insensitive to typical errors; beam loss studies suggest the machine will suffer limited losses even at high currents [6]. Proactive impedance policing has been implemented. Studies of RF system performance [7] and the FEL/RF system interaction 
are underway. Details of these and other beam dynamics studies will be made available in future newsletters.

The machine is presently in the engineering design phase, with many components in procurement. The total project budget is approximately 8.5 $\mathrm{M} \$(\mathrm{US})$. Initial testing of the injector source is underway; initial electron beam commissioning of the driver is expected in October 1997. Ongoing and updated project information is available under the "Technology Transfer" catagory on the Jefferson Lab WWW Home Page (http://www.cebaf.gov/). 


\section{Bibliography}

\section{[1] Reference to Rome FEL paper}

[2] J. B. Flanz and C. P. Sargent, "Operation of an Isochronous Beam Recirculation System", Nuc. Inst. and Meth., A241 (1985), 325-333, North Holland (Amsterdam); J. B. Flanz and C. P. Sargent, "Tests With an Isochronous Recirculation System", IEEE Trans. Nuc. Sci., NS-32, No. 5, October 1985; J. B. Flanz, S. Kowalski, C. P. Sargent, "An Isochronous Beam Recirculation Magnet System", IEEE Trans. Nuc. Sci., NS-28, No. 3, June 1981.

[3] B. Yunn, unpublished.

[4] R. Li, C.L. Bohn, and J. Bisognano, work in progess.

[5] H. Liu, private communication.

[6] R. Li, unpublished.

[7] L. Merminga, "Energy Stability in Recirculating, Energy Recovering Linacs in the Presence of an FEL", to appear in the Proceedings of EPAC 96.

\section{DISCLAIMER}

This report was prepared as an account of work sponsored by an agency of the United States Government. Neither the United States Government nor any agency thereof, nor any of their employees, makes any warranty, express or implied, or assumes any legal liability or responsibility for the accuracy, completeness, or usefulness of any information, apparatus, product, or process disclosed, or represents that its use would not infringe privately owned rights. Reference herein to any specific commercial product, process, or service by trade name, trademark, manufacturer, or otherwise does not necessarily constitute or imply its endorsement, recommendation, or favoring by the United States Government or any agency thereof. The views and opinions of authors expressed herein do not necessarily state or reflect those of the United States Government or any agency thereof. 